\title{
THEORETICAL EFFECT OF A PORTION OF ELECTRICALLY DEAD TISSUE IN THE LEFT VENTRICULAR WALL
}

\author{
BY \\ J. GROSGURIN AND P. W. DUCHOSAL \\ From Centre de Cardiologie, Hôpital Cantonal, University of Geneva, Switzerland
}

Received June 8, 1962

The presence of "electrically dead" myocardial tissue that is inactive during electrical systole is manifested most obviously by a QS wave or simply by a Q wave (Sodi Pallares et al., 1957). However, other abnormalities of the QRS complex may also be due to the presence of small portions of "electrically dead" tissue. Examples would be the development of an rS wave in lead II or notching of the $R$, usually designated as focal block or intraventricular conduction defect.

This problem led us to imagine a "model"* that could link all such graphic expressions. The use of a model permits the study of the theoretical effect of a portion of dead tissue in the electrically active part of the free wall of the left ventricle. Our reasoning can apply only to the outer two-thirds of the free wall of the left ventricle, with regard to what is known about the spread of excitation from the endocardium to the epicardium (Sodi Pallares et al., 1957). The front of excitation is theoretically represented as a polarized membrane or electrical double-layer. This can be replaced by the so-called "equivalent membrane" that is demarcated by the free margin or contour of the polarized membrane itself. A vector, the cardiac vector, can then be substituted for this equivalent membrane (Frank, 1953; Dubouloz, 1953) (Fig. 1). Conduction within the myocardium, with respect to the spread of excitation, progresses in a uniform manner, radially and centrifugally: consequently there is no preferential direction or important variation of speed within the region under consideration. This conforms to present ideas (Scher et al., 1953; Rodriguez, 1960). As it passes the electrically dead tissue, a "hole" is created in the polarized membrane. The margin of this "second hole" forms a second contour in the polarized membrane. This second contour determines, in its turn, another equivalent membrane that may be replaced by a second vector. It is directed toward the endocardium and can be called the "vector of opposition." It modifies the cardiac vector, according to the established rules for the replacement of two linked vectors by one (Grosgurin and Duchosal, 1952).

The modification undergone by the theoretical cardiac vector changes the form of the real vector loop and of the scalar curves. This change is more pronounced when the hole created in the membrane by the dead portion is larger, because the length of the vector of opposition is directly proportional to the surface of this second hole.

The model of dead tissue is within the outer two-thirds of the left anterior region and has a simple geometric form. Two types of models, a sphere and an ellipsoid, will be considered separately (Fig. 2). The electrical consequences will be expressed in vector and in scalar terminology. The sphere creates within the membrane a circular hole of comparatively small dimensions, because it is limited by the width of the wall. The vector of opposition is small, and its existence is brief. The result is an irregularity in the QRS loop and a notching on the QRS complex. This disturbance occurs earlier when the sphere is closer to the endocardium, and vice versa. The ellipsoid chosen

* "Model" is used here to mean a simplified image of reality. The polarized membrane and the cardiac vector are examples of models. Likewise the volume of dead tissue imagined in this discussion is a model that may eventually explain real phenomena, by analogy. 
as a second variant of the model is a flattened sphere. It is an ellipsoid of revolution around its small axis; the long axis is parallel to the wall. Owing to its shape, its size is not too limited by the width of the myocardium: therefore, it can be larger than the sphere and produce a greater circular orifice within the polarized membrane. Necessarily the vector of opposition is larger. If the ellipsoid is situated in the anterior inferior region of the left ventricle near the epicardium, the result is a shift of the major part of the QRS loop toward the head, especially the vectors after 0.02 second. Its scalar translation is an S wave in II and III. This shift is illustrated in Fig. 3 which refers to a case of anterior myocardial infarction.

If the ellipsoid is situated more deeply in the ventricular wall, the result is still an early shift of the QRS loop and a $Q$ wave. If the ellipsoid is very extensive and occupies the outer two-thirds of the wall, it causes an immediate and complete shift of the QRS loop, and a QS wave. Thus, the shifts of the vector loop, and likewise the abnormal QRS complexes of the scalar leads, depend upon the exact position assigned to the ellipsoidal model. This type of reasoning is valid for several regions of the left ventricle, each carrying a particular vectorcardiographic and electrocardiographic pattern.

All the theoretical vectorcardiographic and electrocardiographic modifications mentioned above display an asymmetrical course because the tissue between the obstacle and the epicardium is excited in a different way. The dipoles that constitute the polarized membrane move in a straight line, until they pass the middle of the dead sphere or ellipsoid. To invade the myocardial tissue behind the obstacle, the innermost dipoles then curve inward, as they cannot move

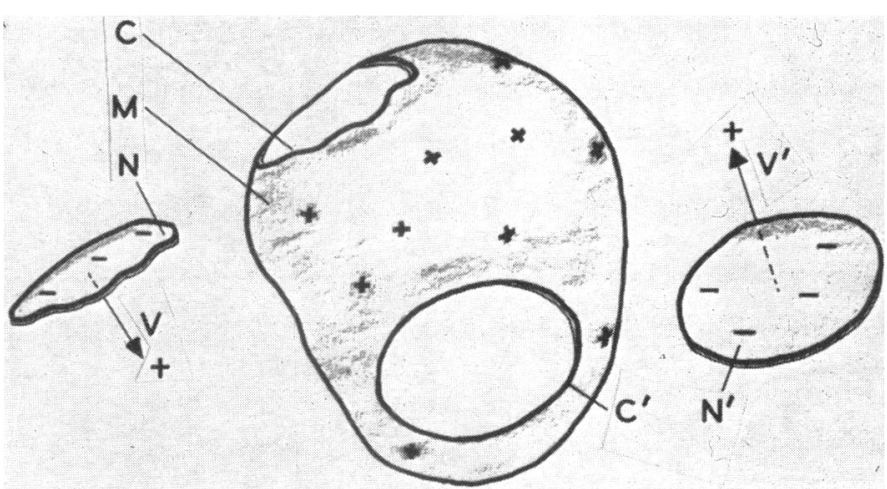

FIG. 1.-M: polarized membrane; + charges outside, - charges inside. C: its "contour" situated at the base of the ventricles. $\mathrm{N}$ : "equivalent membrane" defined by $\mathrm{C} ; \mathrm{N}$ is represented separately; + charges inside, - charges outside relative to $M$. One can substitute $\mathbf{N}$ for $\mathbf{M}$, the vector $\mathbf{V}$ or "cardiac vector" can replace $\mathbf{N}$. If one imagines a "second contour" $C^{\prime}$ caused by an obstacle of dead tissue, the same kind of reasoning applies; one obtains $\mathrm{N}^{\prime}$ then $V^{\prime}$ or the "vector of opposition."
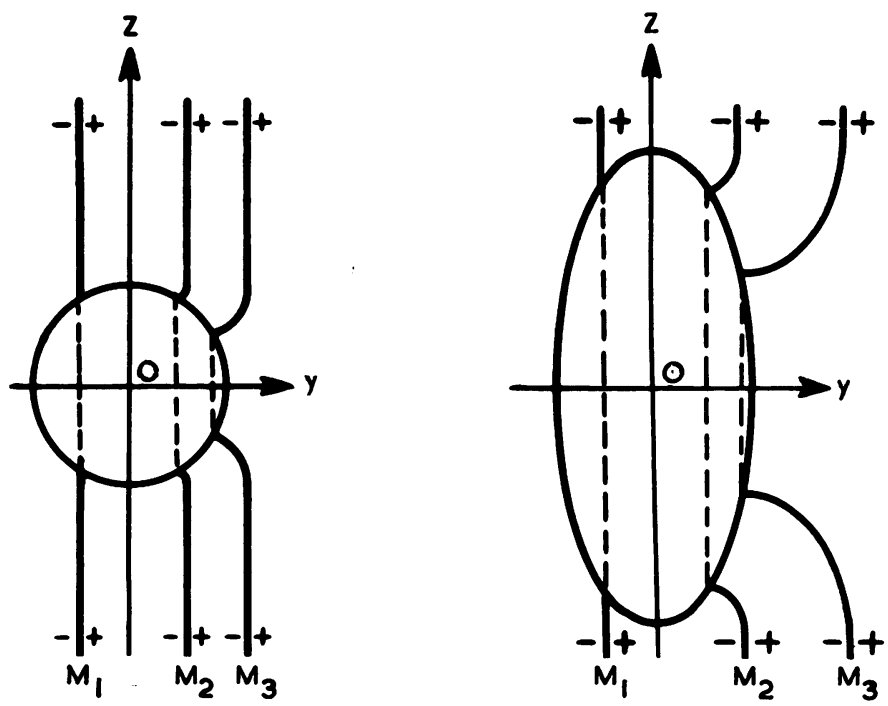

FIG. 2.- Models of dead tissue, theoretically presented within the wall of the left ventricle. A spherical and an ellipsoidal model are considered separately. Their centres are at the origin of rectangular axes $\mathrm{Ox}, \mathrm{Oy}, \mathrm{Oz}$; axis $\mathrm{Oy}$ points toward the epicardium. The ellipsoid revolves around its small axis shown by $O y$, and occupies a circle in the plane $\mathrm{y}=0$ (perpendicular to the figure) $\mathbf{M}_{1}, \mathbf{M}_{2}, \mathbf{M}_{3}$ : successive positions of the polarized membrane which is pierced by an orifice whose position is represented by dotted lines. This is the orifice $\mathrm{C}^{\prime}$ of Fig. 1. On the positive side of $\mathrm{Oy}$, the membrane curves in according to a curve called involute. At any point on this curve the radius of curvature is tangential to the sphere, or to the ellipsoid, respectively. 


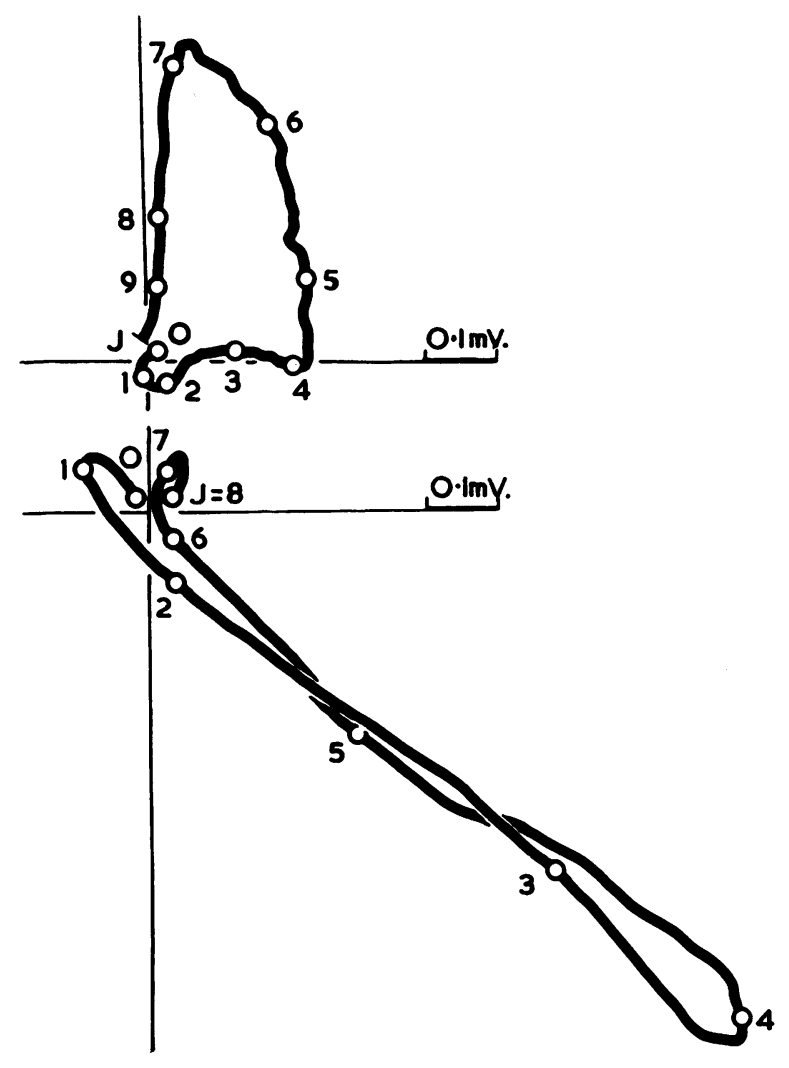

Fig. 3.-Above: QRS loop of the frontal vectorcardiogram in the case of an anterior infarct; the corresponding scalar trace would have rS II-rS III waves. Below: Frontal loop of a normal subject for comparison. The scale of amplification is equal for both vector loops. laterally. Since all dipoles travel at the same speed, those that continue in a straight line reach the epicardium first, and those that have curved reach it later. Therefore, the "second hole" is formed faster than it is closed (Fig. 2). Stated in another way, the modifications undergone by the cardiac vector develop more quickly than they disappear, and an asymmetrical course of events results.

Since the speed of activation is about $30 \mathrm{~cm}$./second under the epicardium (Rodriguez, 1960), the activation of the muscular tissue between the obstacle and the epicardium would be delayed in the order of hundredths of a second and would prolong proportionately the end of the QRS complex.

\section{SUMMARY}

A theoretical study has been presented of the modifications of the QRS loop of the vectorcardiogram produced by some "models" representing a portion of "electrically dead" tissue in the free wall of the left ventricle. In considering the membrane theory, the authors admit that such a model produces a second contour in the polarized membrane. Consequently the effect of the dead tissue can be represented by a vector, the "vector of opposition,"

which modifies the cardiac vector. This study has led to a simple explanation of several vectorcardiographic and electrocardiographic abnormalities. The scalar abnormalities vary from a notch on the $R$ wave to a $Q S$ type wave. The vectorcardiographic abnormalities may cause very important shifts of the whole QRS loop.

\section{REFERENCES}

Dubouloz, P. (1953). Acta cardiol. (Brux.), 8, 489.

Frank, E. (1953). Amer. Heart J., 46, 364.

Grosgurin, J., and Duchosal, P. W. (1952). Acta med. scand., 142, suppl. 266, p. 439.

Rodriguez, M. I. (1960). Inter-American Congress of Cardiology, Rio de Janeiro.

Scher, A. M., Young, A. C., Malmgren, A. L., and Paton, R. R. (1953). Circulat. Res., 1, 539.

Sodi Pallares, D., Bisteni, A., Pileggi, F., and Medrano, G. A. (1957). Princ. cardiol. (Méx.), 4, 163. 